\title{
Infección por Listeria monocytogenes en paciente con sarcoma pélvico
}

Francisco Javier Durán-Garrido', Juan Miguel Gómez-Palomo', Francisco Javier Estades-Rubio', Carlos Jiménez-Garrido', Enrique Nuño-Álvarez ${ }^{2}$, Francisco Javier de Santos-de La Fuente ${ }^{1}$

'Servicio de Cirugía Ortopédica y Traumatología. Hospital Universitario Virgen de La Victoria. Málaga. España

2Unidad de Enfermedades Infecciosas. Hospital Universitario Virgen de La Victoria. Málaga. España

Recibido: 20/04/2020

Aceptado: 24/07/2020

En línea: $31 / 08 / 2020$

Citar como: Durán-Garrido FJ, Gómez-Palomo JM, Estades-Rubio FJ, Jiménez-Garrido C, Nuño-Álvarez E, de Santos-de La Fuente FJ. Infección por Listeria monocytogenes en paciente con sarcoma pélvico. Rev Esp Casos Clin Med Intern (RECCMI). 2020 (Ago); 5(2): 81-83. doi: 10.32818/reccmi.a5n2a9.

Cite this as: Durán-Garrido FJ, Gómez-Palomo JM, Estades-Rubio FJ, Jiménez-Garrido C, Nuño-Álvarez E, de Santos-de La Fuente FJ. Listeria monocytogenes infection in a patient with pelvic sarcoma. Rev Esp Casos Clin Med Intern (RECCMI). 2020 (Ago); 5(2): 81-83. doi: 10.32818/reccmi.a5n2a9.

Autor para correspondencia: Francisco Javier Durán-Garrido. javiduran91@hotmail.com

\begin{tabular}{l} 
Palabras clave \\
\hline$\triangleright$ Listeria monocytogenes \\
$\triangleright$ Infección posquirúrgica \\
$\triangleright$ Inmunodepresión \\
$\triangleright$ Cirugía ortopédica \\
$\triangleright$ Hemipelvectomía
\end{tabular}

\section{Keywords}

$\triangleright$ Listeria monocytogenes

$\triangleright$ Prosthesis-related infections

$\triangleright$ Immunosuppression

$\triangleright$ Orthopedic procedures

$\triangleright$ Hemipelvectomy

\begin{abstract}
Resumen
Listeria monocytogenes es un bacilo grampositivo. La afección en humanos es rara, afectando principalmente a pacientes inmunodeprimidos. No obstante, se ha apreciado un aumento de incidencia reciente. Presentamos el caso de una paciente de 75 años tratada mediante hemipelvectomía y una reconstrucción con una prótesis de anclaje ilíaco, que sufrió posteriormente una infección posquirúrgica por Listeria monocytogenes. Se argumenta el tratamiento seguido y la evolución posoperatoria.
\end{abstract}

La paciente presentó una recuperación completa, con normalización de valores analíticos a los 40 días del alta hospitalaria y 2 años después de la intervención continúa asintomática. Aunque la infección por Listeria monocytogenes tras una cirugía protésica es una complicación infrecuente, es importante tenerla en cuenta y estar preparados para su tratamiento, tanto desde un punto de vista quirúrgico, como para el correcto manejo antibiótico.

\section{Puntos destacados}

$\triangleright$ A pesar de que no sea un germen habitual, debemos considerar Listeria monocytogenes como posible causa de una infección posquirúrgica, especialmente en infecciones larvadas y en pacientes con factores de riesgo.

\section{Introducción}

Listeria monocytogenes es un bacilo grampositivo anaerobio facultativo, que suele encontrarse en aguas residuales, alimentos y diversos animales domésticos. Aunque la infección en el ser humano es poco frecuente, se ha descrito 
Durán-Garrido FJ, Gómez-Palomo JM, Estades-Rubio FJ, Jiménez-Garrido C, Nuño-Álvarez E, de Santos-de La Fuente FJ. Infección por Listeria monocytogenes en paciente con sarcoma pélvico

en relación con válvulas protésicas. La afectación ósea o de prótesis articulares es muy rara; sin embargo, la literatura recoge casos, principalmente en pacientes inmunodeprimidos, ancianos, con enfermedad hepática o renal, o con tumores malignos ${ }^{1}$. Recientemente se ha reportado un aumento de incidencia de esta afección, como la que ha acontecido con el consumo de carne ${ }^{2}$

\section{Caso clínico}

\section{Antecedentes}

Se presenta el caso de una paciente de 75 años, sin ningún antecedente de interés, que no se había sometido a cirugías previas ni poseía hábitos tóxicos Consultó por clínica anodina de dolor lumbo-sacro y fue diagnosticada de hemangioendotelioma epiteloide de alto grado de localización acetabular, con afectación del fondo, columna anterior, posterior e isquion. En el estudio de extensión se descartó enfermedad metastásica.

Tras valorar el caso en un comité multidisciplinar, se decidió llevar a cabo una resección amplia de la hemipelvis y el fémur proximal afectados, con una reconstrucción mediante prótesis de anclaje ilíaco. Antes de la intervención, el Servicio de Radiología Intervencionista realizó una embolización selectiva de los vasos sanguíneos implicados para minimizar el riesgo de sangrado intraoperatorio. Se administró profilaxis antibiótica intravenosa 1 hora antes de comenzar la intervención con cefazolina 2 g, según el protocolo establecido en nuestro centro. El tiempo estimado de cirugía fue de 360 minutos, repitiéndose una dosis antibiótica de $1 \mathrm{~g}$ de cefazolina a los 120 y a los 240 minutos del comienzo. Durante el procedimiento quirúrgico, la paciente requirió una transfusión de 3 concentrados de hematíes. Se pautó antibioterapia profiláctica con cefazolina $1 \mathrm{~g} / 8 \mathrm{~h}$ durante 72 horas posteriores al procedimiento.

\section{Evolución}

A los 14 días de la intervención, la paciente comenzó a presentar fiebre de hasta $38,5^{\circ} \mathrm{C}$. En la analítica de sangre se evidenció un recuento de 23.000 leucocitos/mm y $91 \%$ de neutrófilos.

Se solicitaron radiografía de tórax, hemocultivos, cultivo de orina y tomografía computarizada (TC) toracoabdominopélvica, que pusieron de manifiesto la presencia de una colección periprotésica. Por ello, se decidió realizar una punción ecoguiada (Figura 1) con la extracción de varias muestras con aspecto purulento, que se enviaron a Microbiología para cultivo y antibiograma (Tabla 1).

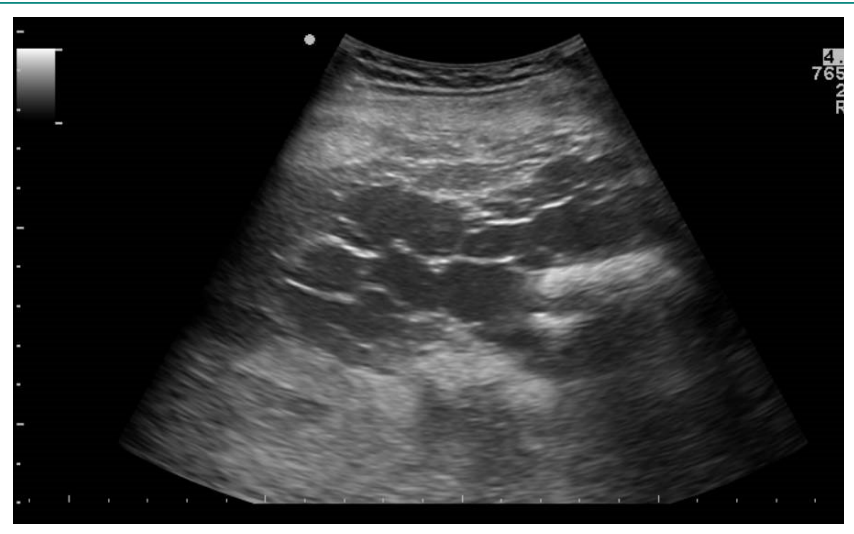

Figura 1. Punción guiada por ecografía de la colección posquirúrgica

\begin{tabular}{|l|c|}
\multicolumn{1}{|c|}{ Antibiótico } & Listeria monocytogenes \\
\hline Penicilina E-Test & $0,25 \mathrm{~S}$ \\
\hline Eritrominicina & $\mathrm{S}$ \\
\hline Ampicilina & $\mathrm{S}$ \\
\hline Meropenem E-Test & $\mathrm{S}$ \\
\hline Trimetoprim-Sulfametoxazol & $\mathrm{S}$ \\
\hline Trimetoprim-sulfametoxazol & $\mathrm{S}$ \\
\hline
\end{tabular}

Tabla 1. Antibiograma

Asimismo, se llevó a cabo una limpieza y desbridamiento quirúrgico, con recambio de componentes móviles, enviados para sonicación. Durante este procedimiento se recogieron hasta seis muestras para cultivo.

Respecto a las muestras extraídas mediante punción ecoguiada, se apreciaron bacilos grampositivos, y el cultivo en agar sangre puso de manifiesto la presencia de Listeria monocytogenes. Posteriormente, los cultivos intraoperatorios confirmaron la infección por Listeria monocytogenes y se pautó antibiótico con gentamicina y ampicilina.

La paciente evolucionó de forma favorable durante el ingreso hospitalario, con una cicatrización adecuada de las heridas y un descenso progresivo de reactantes de fase aguda. La antibioterapia intravenosa se mantuvo hasta completar 30 días de tratamiento, momento en el que se le dio el alta con pauta en domicilio de amoxicilina y trimetoprim-sulfametoxazol durante 12 semanas.

De forma ambulatoria, se incluyó a la paciente en un programa personalizado de rehabilitación, que permitió la reeducación de la marcha. La paciente acudió a revisiones periódicas, presentando una evolución satisfactoria de la herida y los marcadores analíticos; la PCR alcanzó valores compatibles con la normalidad a los 40 días del alta hospitalaria.

Tras 24 meses de evolución, la paciente continuaba asintomática desde el punto de vista infeccioso, deambulando sin ayuda y sin dolor.

\section{Diagnóstico}

Infección aguda posquirúrgica por Listeria monocytogenes.

\section{Discusión y conclusión}

La infección por Listeria monocytogenes es una complicación muy rara en cirugía ortopédica. A pesar de ello, en pacientes inmunodeprimidos o con factores de riesgo, el germen puede alcanzar el torrente sanguíneo a través del sistema linfático y producir una infección protésica ${ }^{3}$. En cirugía protésica, debemos diferenciar la infección aguda, como en nuestra paciente, que aparece en el primer mes tras la cirugía, de la infección subaguda o crónica ${ }^{4}$. Por lo general, las infecciones agudas y subagudas se adquieren en el período perioperatorio, mientras que las infecciones crónicas suelen relacionarse con la vía hematógena.

La infección aguda suele manifestarse con dolor y tumefacción articular de aparición súbita, así como con la presencia de fiebre y malestar general. Por el contrario, la infección crónica suele causar dolor subagudo, presencia de fístula e incluso aflojamiento de los componentes protésicos.

La infección protésica por listeriosis en el ámbito de la cirugía ortopédica tiene una incidencia muy escasa, y la mayoría de los casos reportados son 
pacientes con artroplastia de cadera ${ }^{1,5}$. Nuestro caso es el primero reportado en la literatura científica de infección protésica por Listeria monocytogenes en una paciente con sarcoma pélvico tratado mediante hemipelvectomía y prótesis de reconstrucción.

La infección por Listeria monocytogenes suele ser menos agresiva que la de los gérmenes habituales, lo que puede conducir a un retraso en el diagnóstico y, por consiguiente, en su tratamiento. Otro motivo que puede dificultar el diagnóstico es la capacidad que posee Listeria monocytogenes de crear un biofilm, que necesita usar procedimientos específicos, tales como la sonicación o determinados compuestos químicos, que pueden ayudar a solucionar este problema ${ }^{6}$

Por regla general, Listeria monocytogenes suele tener sensibilidad in vitro a diversos antibióticos, siendo el fármaco de elección la ampicilina o la penicilina G No obstante, el retraso en la acción de estos antibióticos hace que, en determinados pacientes, como el caso presentado, sea necesario asociar una pauta con gentamicina para obtener una sinergia ${ }^{7.8}$. En pacientes alérgicos se puede sustituir por trimetoprim-sulfametoxazol. La pauta antibiótica debe ser prolongada, sobre todo en pacientes inmunocomprometidos ${ }^{9}$, con un tratamiento de al menos 3 semanas con antibioterapia intravenosa, seguido de hasta 3 meses con antibioterapia oral.

En el caso que nos atañe, encontramos diversos factores que incrementaban el riesgo de infección, como la reconstrucción mediante prótesis, que aumenta el riesgo de infección con respecto a aquellos pacientes donde únicamente se realiza una resección amplia ${ }^{10}$. Por otro lado, también se ha relacionado con un incremento en el riesgo de infección posoperatoria la necesidad de transfusiones ${ }^{11,12} \mathrm{O}$ una duración prolongada de la cirugía.

Podemos concluir que, aunque la infección de prótesis articular por Listeria monocytogenes es muy infrecuente, hay que tenerla en cuenta en pacientes con factores de riesgo, incluso en casos con cultivos negativos, precisando en ocasiones la utilización de herramientas diagnósticas adicionales como la reacción en cadena de la polimerasa (PCR), determinados test químicos o la sonicación de los implantes. En ocasiones, su presentación clínica es poco agresiva, lo que puede dificultar la realización de un diagnóstico y su tratamiento precoz.

\section{Bibliografía}

1. Allerberger F, Kasten MJ, Cockerill FR 3rd, Krismer M, Dierich MP. Listeria monocytogenes infection in prosthetic joints. Int Orthop. 1992; 16(3): 237-239. doi: 10.1007/bf00182702.

2. Lepe JA. Current aspects of listeriosis. Med Clin (Barc). 2020 Jun 12: 154(11): 453-458. doi: 10.1016/j.medcli.2020.02.001.

3. Mook P, O'Brien SJ, Gillespie IA. Concurrent conditions and human listeriosis, England, 1999-2009. Emerg Infect Dis. 2011; 17(1): 38-43. doi: 10.3201/ eid1701.101174.

4. Schafroth M, Zimmerli W, Brunazzi M, Ochsner PE. Infections. In: Ochsner PE (ed.). Total Hip Replacement. Berlin, Heidelberg. Springer, 2003. Accesible en: https://link.springer.com/chapter/10.1007/978-3-642-55679-1_5 (último acceso abril 2020).

5. Cone LA, Fitzmorris AO, Hirschberg JM. Is Listeria monocytogenes an important pathogen for prosthetic joints? J Clin Rheumatol. 2001; 7(1): 3437. doi: 10.1097/00124743-200102000-00008.

6. Drago L, Signori V, De Vecchi E, Vassena C, Palazzi E, Cappelletti L, et al. Use of dithiothreitol to improve the diagnosis of prosthetic joint infections. J Orthop Res. 2013; 31(11): 1694-1699. doi: 10.1002/jor.22423.

7. Drevets DA, Canono BP, Leenen PJ, Campbell PA. Gentamicin kills intracellular Listeria monocytogenes. Infect Immun. 1994; 62(6): 2222-2228.

8. Moellering RC Jr, Medoff G, Leech I, Wennersten C, Kunz LJ. Antibiotic synergism against Listeria monocytogenes. Antimicrob Agents Chemother. 1972; 1(1): 30-34

9. Massarotti EM, Dinerman H. Septic arthritis due to Listeria monocytogenes: report and review of the literature. J Rheumatol. 1990; 17(1): 111-113.

10. Angelini A, Drago G, Trovarelli G, Calabrò T, Ruggieri P. Infection after surgical resection for pelvic bone tumors: an analysis of 270 patients from one institution. Clin OrthopRelat Res. 2014; 472(1): 349-359. doi: 10.1007/s11999-013-3250-x.

11. Tornero E, Pereira A, Basora M, Lozano L, Morata L, Muñoz-Mahammud E, et al. Intraoperative transfusion of red blood cell units stored $>14$ days is associated with an increased risk of prosthetic joint infection. J Bone Jt Infect. 2019; 4(2): 85-91. doi: 10.7150/jbji.30001.

12. Ercole FF, Chianca TC, Duarte D, Starling CE, Carneiro M. Surgical site infection in patients submitted to orthopedic surgery: the NNIS risk index and risk prediction. Rev Lat Am Enfermagem. 2011; 19(2): 269-276. doi: 10.1590/s0104-1169201100000007. 